\title{
Design and Simulation of Storage Location Optimization Module in AS/RS Based on FLEXSIM
}

\author{
Guiliang Zhou \\ Huaiyin Institute of Technology, Huaian , China \\ Email: yessuccess@163.com \\ Lina Mao \\ Huaiyin Institute of Technology, Huaian , China \\ Email: 30721409@qq.com
}

\begin{abstract}
This paper firstly points out the importance of the storage location optimization in Automated Storage and Retrieval Systems (AS/RS). Then it has analyzed the basic situation of the current storage location assignment strategy, and indicated the deficiency of the storage location selection under the current strategy. Afterwards, it has designed the storage location optimization module in AS/RS. And then it has constructed the mathematical model on the storage location assignment in AS/RS, and then optimized and solved it, performed instance simulation design and analysis on the storage location assignment by means of Flexsim. Finally, it has concluded that the storage location assignment model after optimization would solve the storage location assignment problem in AS/RS effectively and improve the loading-unloading efficiency, as will provide technical support for the enterprise to improve efficiency and reduce cost.
\end{abstract}

Index Terms-AS/RS, storage location assignment optimization, FLEXSIM simulation, energy consumption model

\section{INTRODUCTION}

The Automated Storage and Retrieval Systems (AS/RS) is a modern logistics facility which gathers storage, handling, transportation and distribution in integral whole. It is the important part of the plant logistics system, computer integrated manufacturing system (CIMS) and the flexible manufacturing system (FMS) and represents the developing trend of the logistics. AS an important part of the modern logistics system, AS/RS is a multilayer elevated warehouse system. It has integrated computer information management and the equipment control. The high efficiency of the AS/RS is based on efficient storage location management. To manage the storage location is to assign and use the storage location reasonably. We need not only to consider how to improve the utilization of the storage location, but also to guarantee the unloading efficiency. Storage location assignment includes two aspects: one is to assign the optimum storage location for the materials to be loaded, which is loading storage location assignment; the other is to select the storage location of materials to be unloaded.
In order to manage the AS/RS much more effectively, thus to improve the whole operation efficiency, we need to optimize the storage location so as to decrease the cost on cargo handling and storing, reduce the overstock capital and time consumption during the processes of storing and handling, therefore reduce the logistics cost and increase the profit. Just basing on this thought, the paper optimizes and simulates the storage location assignment combined with Flexsim logistics simulation software and so as to provide theoretical basis for improving the loading-unloading efficiency.

\section{STORAGE LOCATION ASSIGNMENT STRATEGY}

For advancing the efficiency of the AS/RS and shortening the moving distance of the stacker, the ware location assignment strategy commonly adopted are such as uniform shelf load, light upper layer and heavy lower layer; accelerating the turnover, first in and first out; improving the reliability, division laneway storage; advancing efficiency, nearest loading-unloading; product correlation, etc. 1) Balance the workload of each workaround. To distribute the goods need large orderpicking volume to different picking areas equally, and this would balance the order-picking workload of each area and avoid congestion in some area. 2) To storage the goods with high loading-unloading frequency near to the accesses, and this is beneficial to accelerating the loading-unloading operation and improving the loadingunloading efficiency. 3) Convenient replenishment. It may cut down the labor quantity during the replenishment process significantly that to decide the storage and retrieval position according to the dimensions of containers and pallets. 4) Product correlation principle. The goods with close correlation are usually loaded or unloaded at the same time. So they should be storied at adjacent positions as much as possible to shorten the picking distance. 5) Modified first-in/first-out algorithm. In order to prevent the goods from excessive long time storage, we can set up upper limit for storage time. According to the modified first-in/first-out principle, for the goods exceed the upper limit, the principle should be strictly implemented, that is the early loading goods is 
prior to unload; for the goods within the upper limit, the principle may be ignored to some degree, they can be unloaded according to the nearest distance principle.

\section{PROBLEMS IN STORAGE LOCATION SELECTING IN} AS/RS

Regarding to the several principals above, there are certain deficiencies in application. Random storage will lead to adjacent storage of many conflicting cargoes such as the chemical material and food, the tea and camphor ball, etc... This would easily make the cargoes metamorphic and damaged. Though classification storage avoids such problem well, once the division is not adapt to the actual arriving cargo volume, it will engender that some subareas are idle and the others are strained, which would reduce the turnover and utilization efficiency of the storage location substantially and cause resources to be wasted.

In addition, there are still many companies adopting the traditional sequence numbering mode according to layers or columns [1]. Though the storage location numbers are sequential in certain layer or column, the step points will emerge at the end of layers or columns. The higher the shelf is, or the deeper the laneway is, the greater the step is. Furthermore, the storage location numbering mode does not consider that the cargoes should be put near to the origin as much as possible. Also, the warehousing frequency is not taken into account. For the hysteresis nature of storage location optimization, it can judge whether the previous selection is optimal or not only when cargoes arrive again after the arriving cargoes are stored. So such model can be used in the warehouses which have already finished division in accordance with classification. Once the cargoes arrive with rich varieties, randomness and large amounts, the optimizing results will quite differentiate from the actual optimum.

The problems in storage location selecting can be shown as Figure 1.

Basing on the analyses on the deficiencies of the methods above, the paper has constructed the mathematical model on the storage location optimizing in accordance with the principle of minimum storage energy consumption. We have carried out statistical analysis on the loading-unloading energy consumption data for goods of unit mass, and optimized the numbering on the storage location in warehouse, then gotten the optimizing storage mode. The method to construct storage location intelligent selection module based on the database of the warehouse and the optimized energy consumption model can effectively solve the problems in current storage mode in AS/RS.

\section{The Intelligent Decision Module Design on STORAGE LOCATION ASSIGNMENT IN AS/RS}

\section{A. Environment and flow of the intelligent decision module design}

The paper takes the AS/RS of Jiangsu Feiliks International Logistics Inc. for example to study. The warehouses of Feiliks contain general warehouse, constant temperature warehouse and high-value warehouse. The functional areas of warehouses are divided into receiving area, pending disposal area and dispatch area, etc. The area of general warehouse is 26,000 square meters; the shelves are standardized 20 floors entirely, with 29,500 storage locations. The handling operation equipments are consists of 7 sets of forklifts and stackers respectively. It is the background and basis to design the intelligent decision module. The module has taken Microsoft Access 2000 as the backed database, the advanced language Microsoft Visual Basic6.0 as the front-end development tool. It has integrated the optimization simulation model with GA optimization algorithm and Flexsim simulation platform. The design has adopted the object oriented idea and made full use of the flexibility and reliability of the Intelligent Decision Support System (IDSS). The design flow chart of the module is shown as figure 2 .

\section{B. The database management system of the AS/RS}

The database system is a computer application system. Besides the database taking data as the mainbody, DBMS — the system software to manage the database, it also includes the computer hardware environment and operating system environment to support the database system, as well as the men to manage and use the database system. As is shown in Figure 3.

The DBMS of this system is Microsoft Access 2000. Microsoft Access 2000 has powerful function and simple operation. It can offer us lots of convenient visual operation tools (form-builder, query designer, report designer, database access page designer, etc...) and wizards (database wizard, form wizard, query wizard, report wizard, etc...). By using them, we can construct the database management module and build a simple management information system (see Figure 4).

\section{CONSTRUCTION, OPTIMIZATION AND SOLUTION OF THE MATHEMATICAL MODEL ON THE STORAGE LOCATION ASSIGNMENT IN AS/RS}

Firstly, we construct the initial energy consumption model, and then optimize it properly. We number the storage location according to the concrete conditions of the energy consumption so as to select the storage location adapt to the goods to be loaded and unloaded, and thus reduce the operation cost and improve the efficiency of each link [2].

\section{A. The energy consumption mathematical model on the loading-unloading operation of a single material}

Normally, energy consumption is related to the storage position of the goods, loading-unloading frequency and weight of the goods. Therefore, the total loadingunloading energy consumption of some goods could be expressed as:

$$
E=K \times M \times N \times d(h, L)
$$

In which:

$\mathrm{E}$ is the total loading-unloading energy consumption /J; 
$\mathrm{K}$ is the scale factor, the value of which lies in $1.0 \sim$ 1.5 ;

$\mathrm{M}$ is the weight of goods $/ \mathrm{kg}$;

$\mathrm{N}$ is the loading-unloading frequency of goods within the statistical time;

$d(h, L)$ is the energy consumption function which is related to the position of the storage location for per unit weight $/(\mathrm{J} \cdot \mathrm{kg}-1)$.

According to the analysis of the actual situation, when loading the material to a certain storage location, the energy consumption for per unit weight can be expressed as:

$d(h, L)=g \times h+f \times g \times L+a \times x$

In which:

$g$ is the acceleration of gravity/(m $\bullet$ s-2);

$a$ is the horizontal acceleration of the conveyor/(m $\bullet$ s2);

$h$ is the minimum rising height for storing the goods to a certain layer $/ \mathrm{m}$;

$L$ is the horizontal distance from the storage position of the goods to the loading platform $/ \mathrm{m}$;

$x$ is the horizontal delivery distance of the conveyor from the warehouse access to the loading platform /m;

$f$ is the friction coefficient between the AGV and track, the value of which lies in $0.1 \sim 0.3$;

The formula (1) and formula (2) have shown that, the loading-unloading energy consumption of certain goods is not only related to the weight and loading-unloading frequency, but also related to the storage position in AS/RS and the delivery distance of conveyor belt [3]. With a given weight and loading-unloading frequency, the energy consumption differs with different storage position. This is the realistic foundation to carry out the storage location optimization for AS/RS.

\section{B. Optimization of the storage location mathematical model}

According to the principle that there exists minimum energy consumption for goods storing, combined with formula (1) and (2), we can get the integral optimization mathematical model on the storage location in AS/RS.

$$
\left\{\begin{array}{l}
\min E(h, L)=\sum_{i} K \times M_{i} \times N_{i} \times d_{i}(h, L) \\
d_{i}(h, L)=g \times h_{i}+f \times g \times L_{i}+a \times x_{i}
\end{array}\right.
$$

In which:

$E(h, L)$ is the summation of loading-unloading energy consumption for the whole material in AS/RS/kJ;

$d_{i}(h, L)$ is the energy consumption function for per unit weight when loading a certain storage location/(kJ • kg1);

$M_{i}$ is the weight of goods in a certain storage location/kg; $N_{i}$ is the loading-unloading frequency of goods in a certain storage location within the statistical time; $h_{i}$ is the height between the storage location and the loading platform, which is related to the number of layers $/ \mathrm{m}$;

$L_{i}$ is the horizontal distance between the storage location and the loading platform, which is related to the number of columns/m;

$i$ is the number of storage location, which can but only take value of $1,2,3 \cdots \cdots$;

$x_{i}$ is the distance for the goods in storage location $i$ to travel on the conveyor $/ \mathrm{m}$.

From the mathematical model, we can get that the total loading-unloading energy consumption for the whole material in AS/RS is related to the total statistical weight of the whole material (the product of the weight and the loading-unloading frequency of the material) and the energy consumption function $d_{i}(h, L)$ of the storage location for per unit weight remarkably. The bigger the statistical weight of the material is, the more the loadingunloading energy consumption is; and the bigger the energy consumption function of the storage location for per unit, the more the loading-unloading energy consumption is. According to the storage location optimization model, we can conclude that in order to get minimum value of the total loading-unloading energy consumption $\mathrm{E}$, it is necessary to store the material with the biggest statistical weight $(\mathrm{M} \times \mathrm{N})$ into the storage location with the lowest energy consumption for per unit weight.

\section{Solution of the model}

We take a certain AS/RS of the Jiangsu Feiliks International Logistics Inc. for example to solve the model. The warehouse has 5 double-face shelves which can be divided into 6 layers and 40 columns. There are 240 lattices in total. The specification of each lattice is $1.0 \times 1.0 \times 1.0$. The speeds of the stacker are: $v_{x}=10 \mathrm{~m} / \mathrm{s}$, $v_{y}=3 \mathrm{~m} / \mathrm{s}$. The layout of the warehouse is shown as Figure 5.

Traditionally, the storage location is column based numbered as is shown as figure 6. Goods is stored in sequence according to the traditional storage location number from small to big. Thus it is hard to avoid some unreasonable situations such as certain commonly used goods is stored in the furthest position of the shelves or heavier material is stored in the higher shelves. Therefore, the loading-unloading efficiency of goods and the stability of the shelves would be affected. Meanwhile, the operating cost of AS/RS would rise and the loadingunloading speed would decrease [4].

Aiming at this situation, the paper has presented optimized storage location arrangement diagram in accordance with the principle of minimum energy consumption for loading-unloading material (shown as Figure 7), and constructed the mathematical model on storage location optimization. 


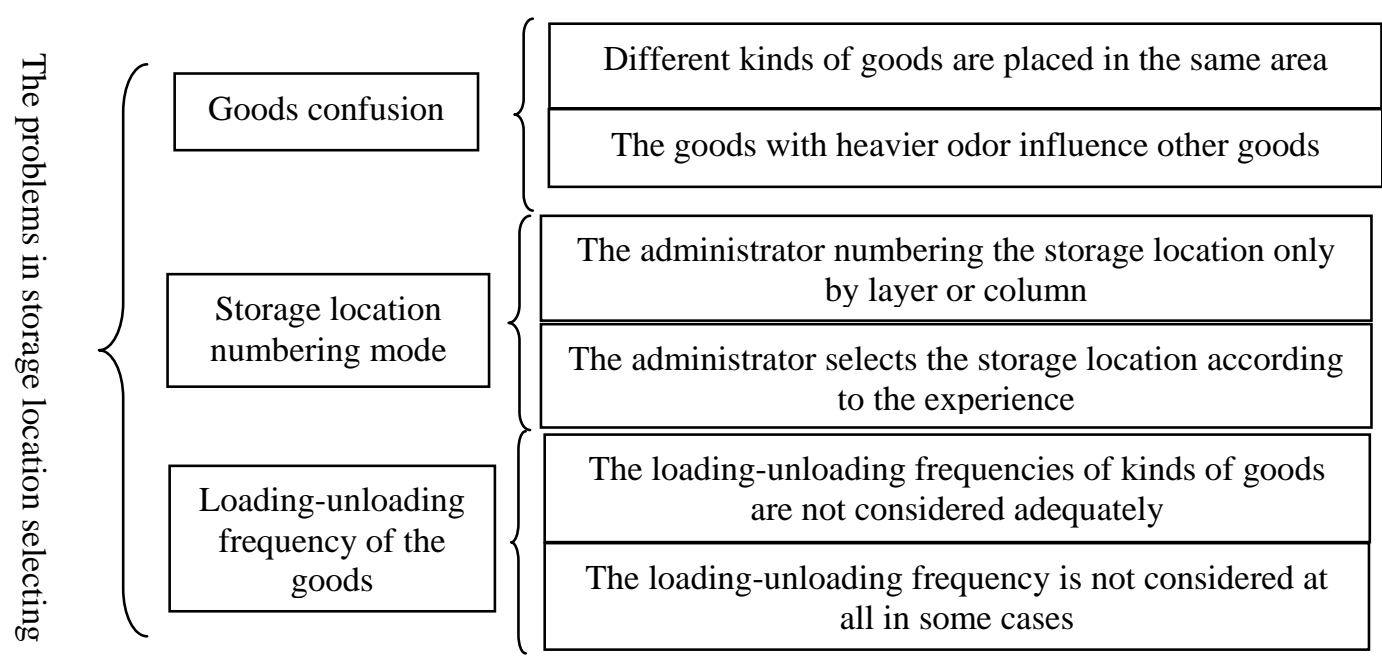

Figure 1. The problems in storage location selecting

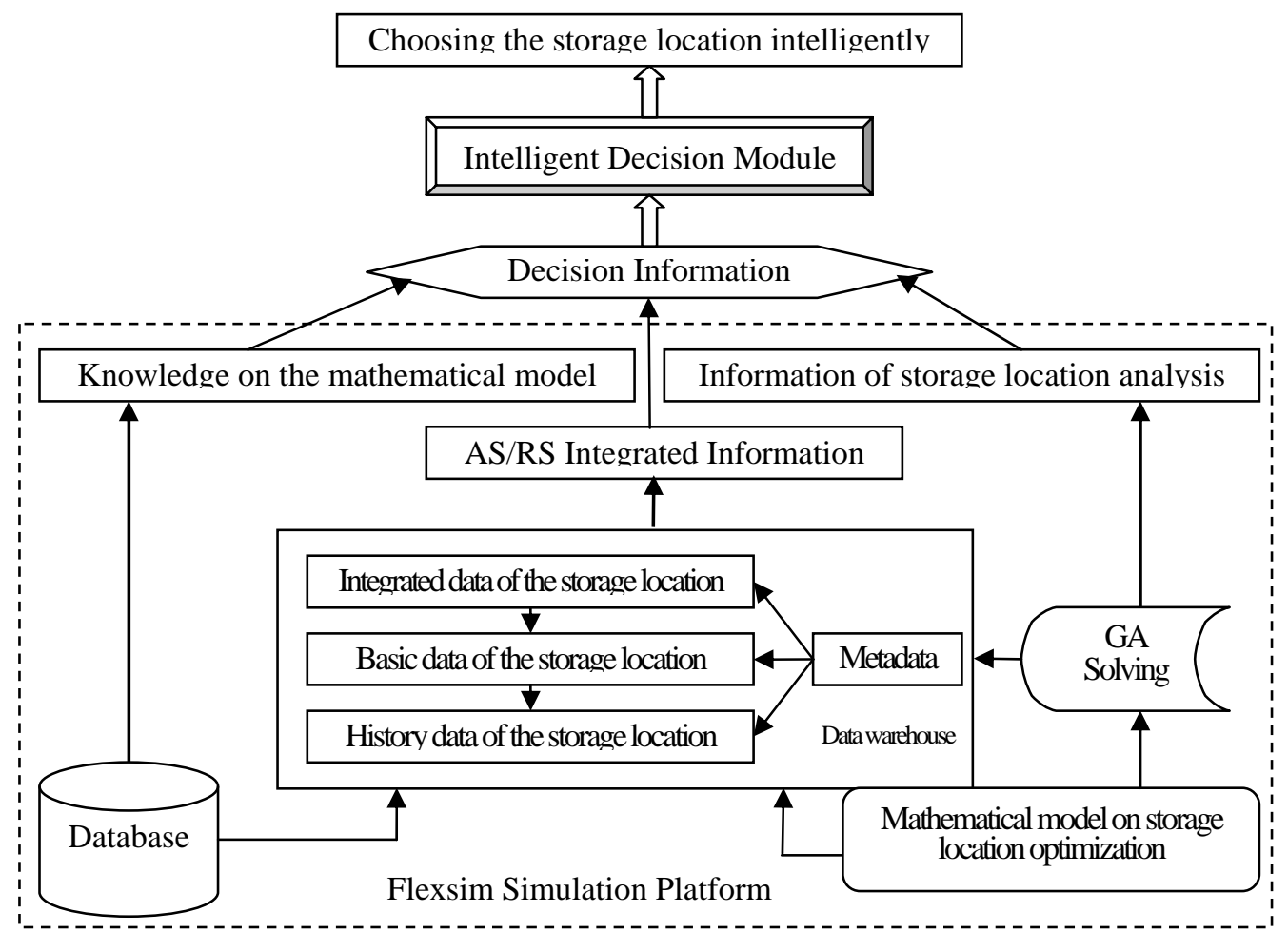

Figure 2. Design flow chart of the module 


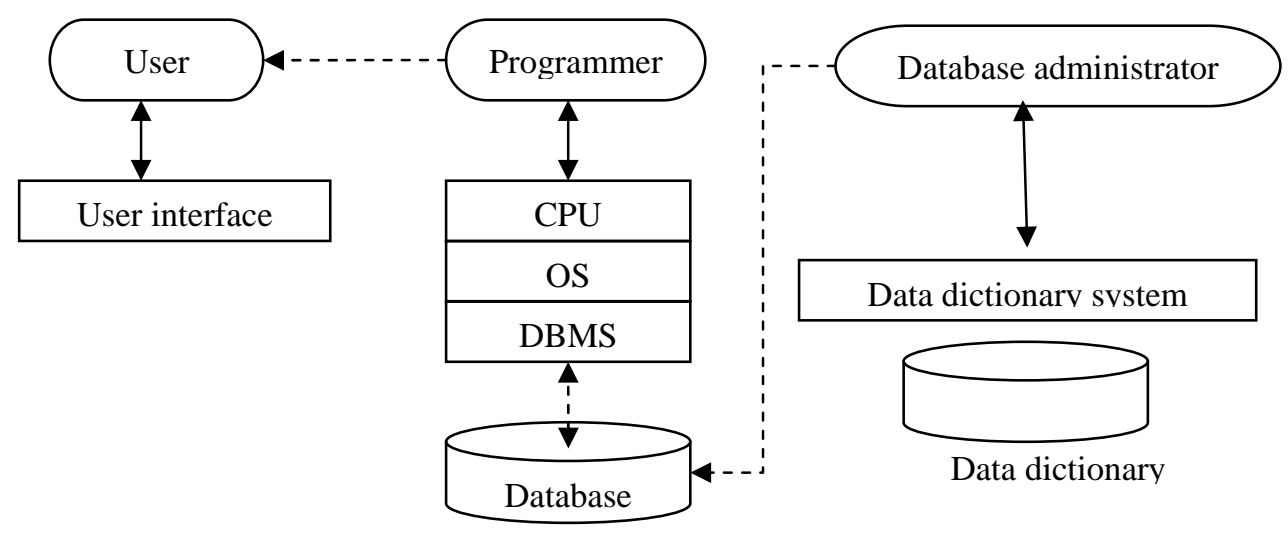

Figure 3. The schematic diagram of database system

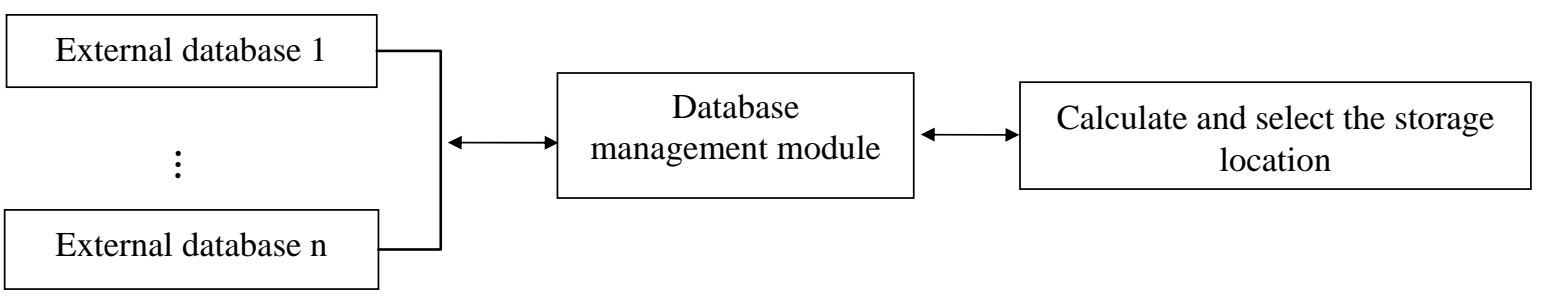

Figure 4. The management structure diagram of the database

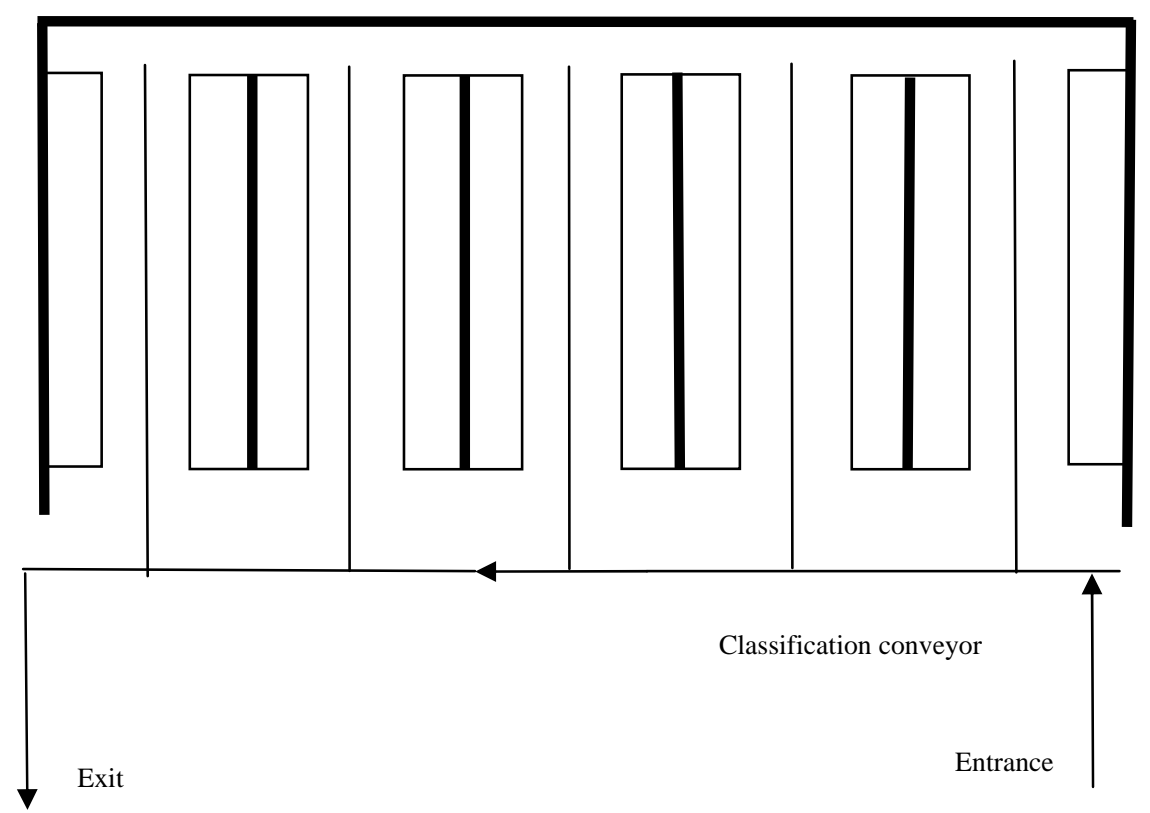

Figure 5. The layout of the warehouse

As would solve the problems exist in current storage mode effectively, reduce the operating cost of AS/RS and improve the loading-unloading efficiency.

According to related principles, it consumes less energy to store the same material in horizontal direction than in vertical direction. Therefore, the storage location number in horizontal direction should be considered firstly when loading and unloading the material in AS/RS.

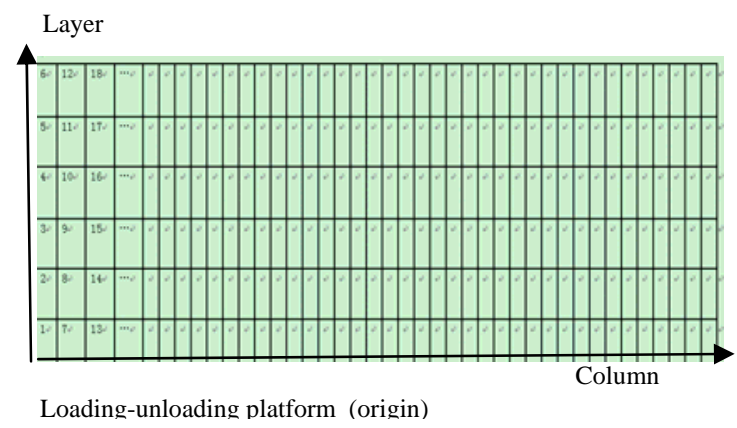

Figure 6. The traditional storage location arrangement diagram 


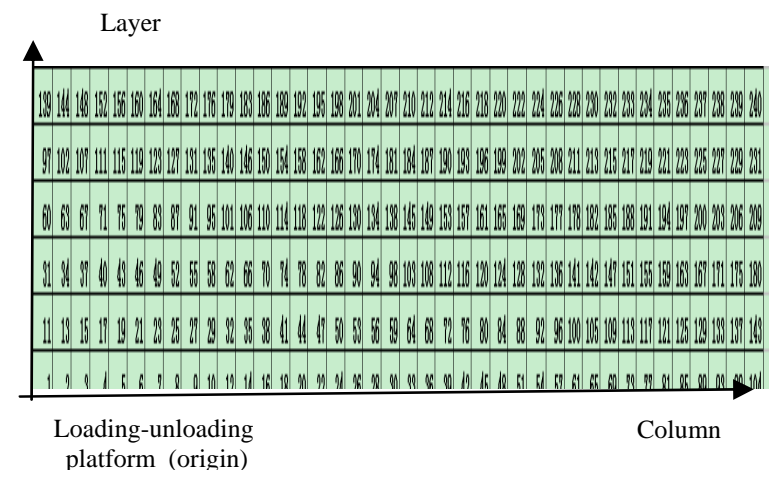

Figure 7. The optimized storage location number arrangement diagram

By using the Microsoft Access 2000 to build the database, we stat and analyze the materials in AS/RS and choose the representative ones, then simulate the loadingunloading energy and time consumption of 10 batch of material in AS/RS randomly under the two different storage modes. Calculating the data by computer and analyzing them by Microsoft Excel 2003, we can see the outcome shown as Figure 8 and Figure 9.

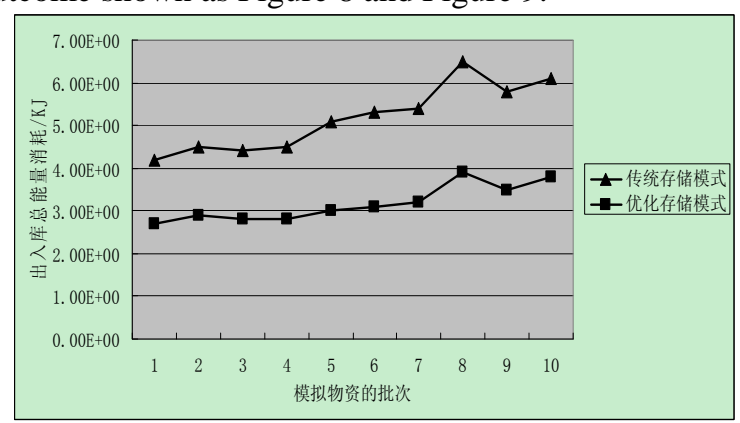

Figure 8. The energy consumption comparison of different storage mode

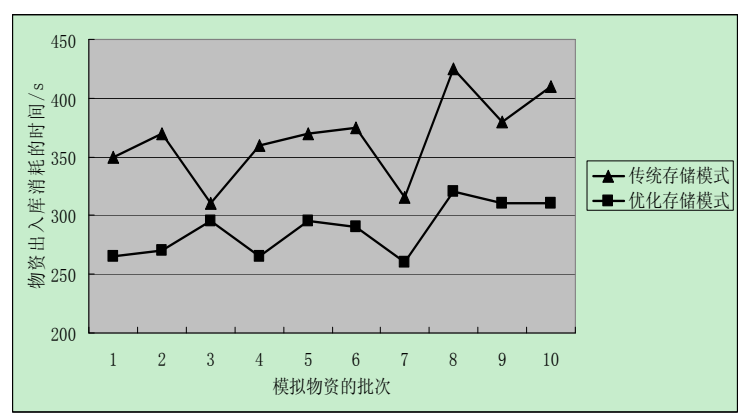

Figure 9. The time consumption comparison of different storage mode

Figure 8 and Figure 9 indicate that the energy and time consumption of storage is much lower than the traditional storage mode after optimization. Thus it can be seen that it can save the energy and time consumption, reduce the operating cost of AS/RS and improve the loadingunloading efficiency when applying the optimized storage location numbering and storage location optimization model.

\section{OPTIMIZATION SIMULATION AND VERIFICATION ON STORAGE LOCATION ASSIGNMENT BASED ON FLEXSIM}

\section{A. The basic objects based on Flexsim modeling}

Flexsim offers a series of basic objects to construct the system simulation model. The objects in this module are mainly divided into four categories. 1) Fixed Resources Object. The Fixed Resource objects are the main object of this simulation model. They determine the process of the model. It mainly includes the objects such as Source, Queue, Processor, Sink, Combiner, Separator, MultiProcessor, Conveyor, Merge Sort, Flow Node, Rack and Reservoir, et.. 2) Task Executer Object. The Task Executer objects can be obtained from the Fixed Resource objects and implement task such as material handling or production operation and so on. A Task Executer object can assign tasks to the other Task Executer objects, or manage all the Task Executer objects in the model. The Task Executer objects are not involved in the process assignment in the model. It mainly includes the objects such as Dispatcher, Operator, Transporter, Elevator, Robot, Crane and ASRS vehicle and so on. 3) Node Object. The Network Node objects are used to set the action routes for the Task Executer objects. It mainly includes the objects such as Network Node and Traffic Control and so on. 4) Visual Object. The Visual Objects used in the simulation model display various information, marks, pictures and charts and so on. The Visual Tool and Recorder objects are used to improve the visual impression of the simulation model, and meanwhile display and collect the output data of the model at the real time. It mainly includes the objects such as Visual Tool and Recorder and so on.

\section{B. Equations Design of the traditional storage mode}

The template is designed so that author affiliations are not repeated each time for multiple authors of the same affiliation. Please keep your affiliations as succinct as possible (for example, do not differentiate among departments of the same organization). This template was designed for two affiliations.

In the traditional mode, the running interface of the storage location selection module based on Flexsim is shown as Figure 10. The different color stands for different materials, but the materials entering the same conveyor belong to the same kind. Sorting conveyors are respectively 2 meters, 9 meters, 16 meters, 23 meters, 30 meters away from the entrance to assign loading. The shelves are supposed to have 10 rows with 40 columns and 6 layers in each row. The parameters of shelf and tracker are shown as Figure 11 and Figure 12. 


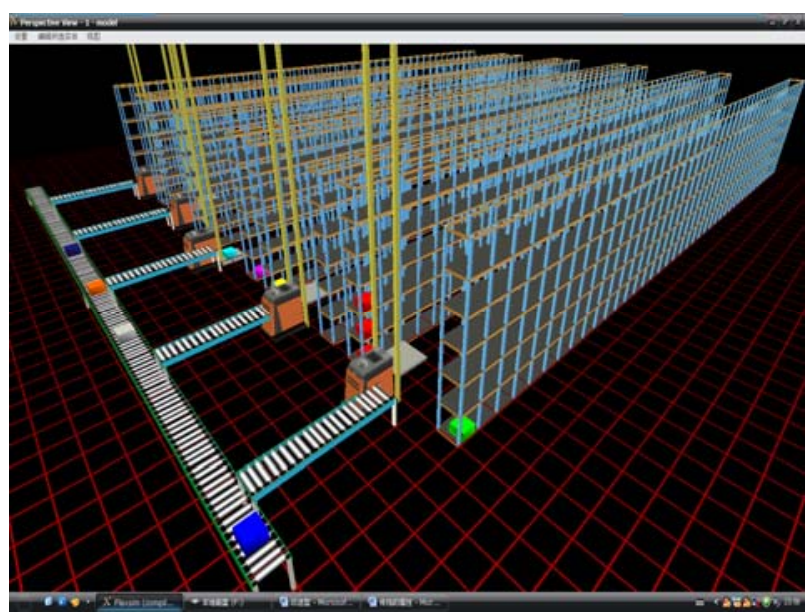

Figure 10. The running interface of the traditional mode

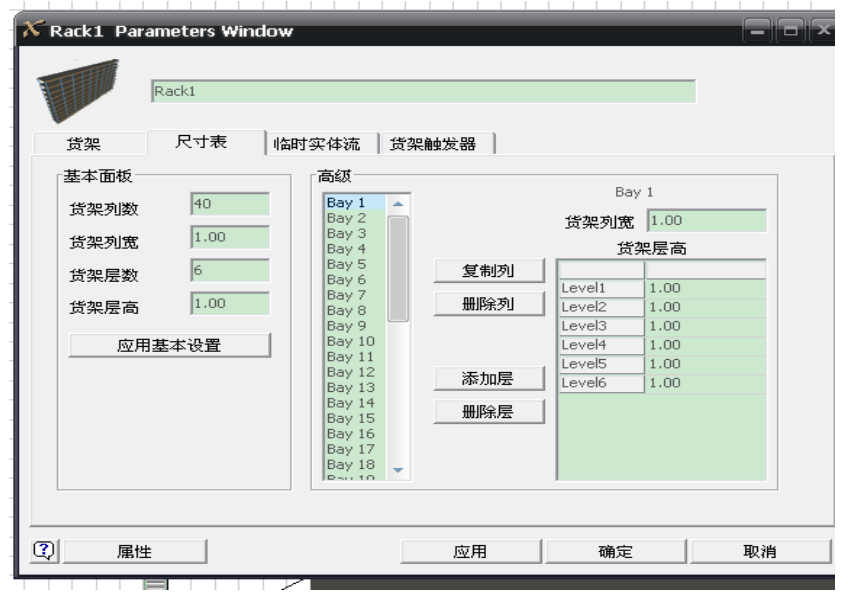

Figure11. The parameter setting for the shelf attribute

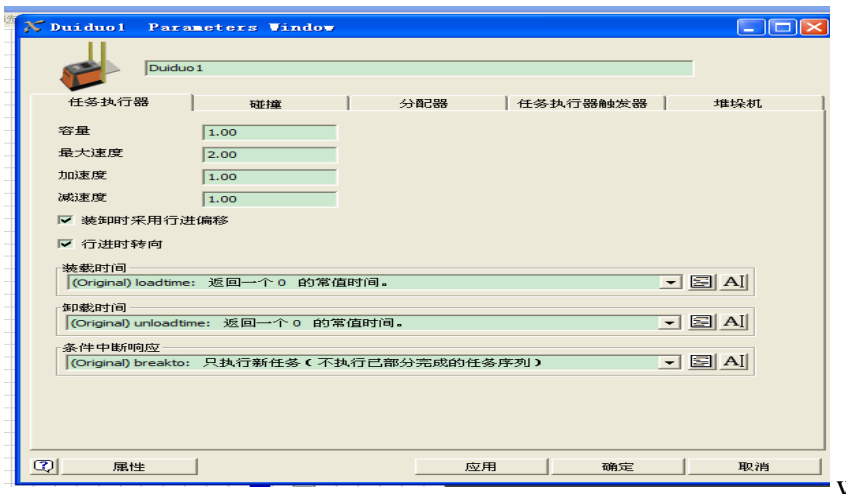

Figure 12. The parameter setting for the stacker

\section{Design of the optimization storage mode}

On the premise of minimum storage location energy consumption, we optimize the storage location number arrangement by using the Flexsim simulation, and the running interface is shown as Figure 13. The optimized model mainly chooses 4 rows shelves with 10 columns and 10 layers each row [5]. According to the specific conditions of material, the stacker will select the low energy consumption storage location for the high energy consumption material. The attribute setting of the shelf and stacker are shown as Figure 14 and Figure 15.

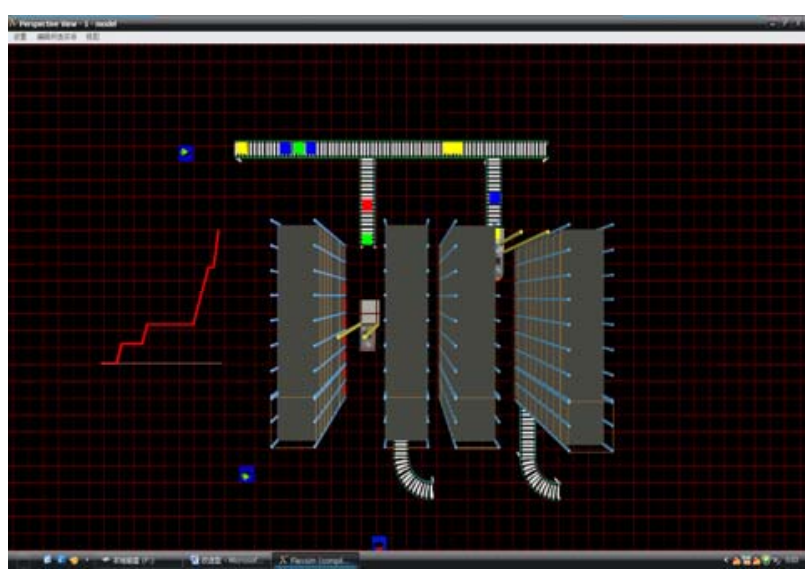

Figure 13. The running interface of the optimization mode

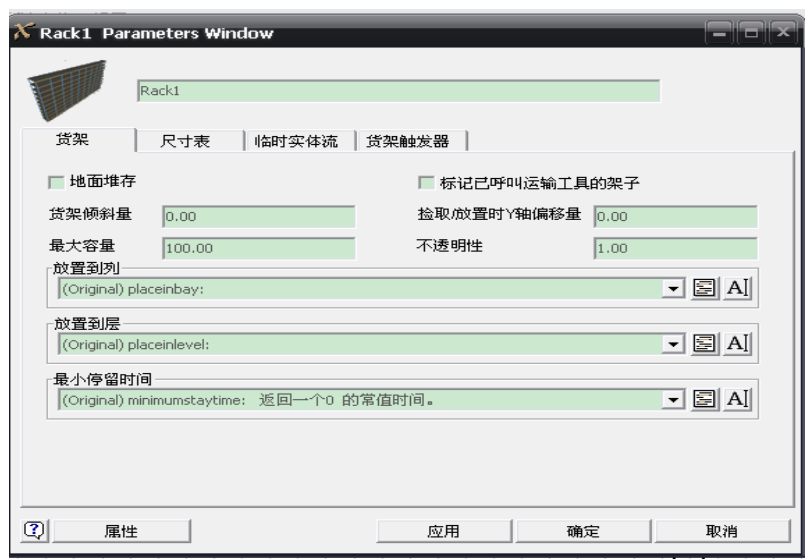

Figure 14. The shelf attribute setting

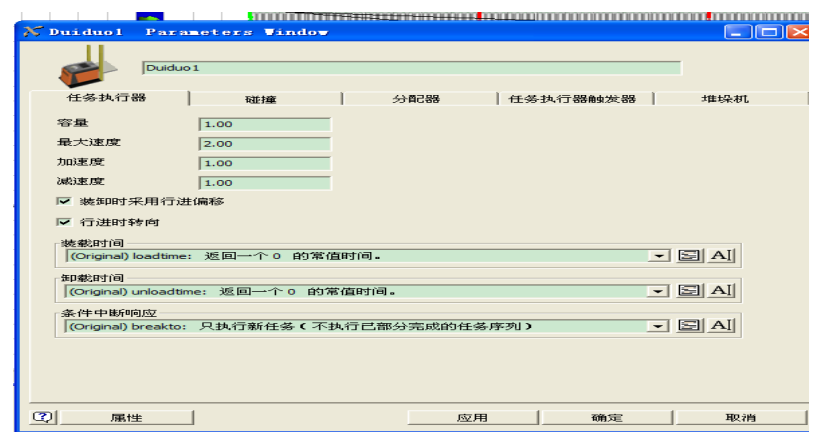

Figure15. The stacker attribute setting

After running the simulation optimization on Flexsim, we can see the storage location quantity curve of a shelf which is shown as Figure 16. And the contrast result of the simulation data output of the unloading buffer area before and after the system improved is shown as Table 1. 


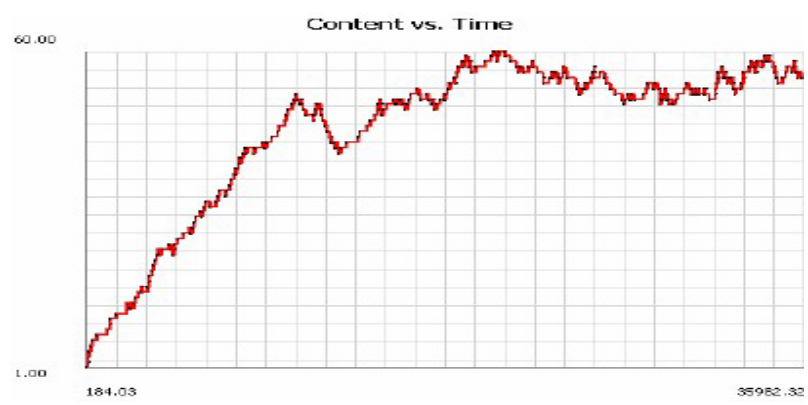

Figure16. Storage location quantity curve of certain shelf after simulation

TABLE I. SIMULATION DATA OUTPUT OF THE UNLOADING BUFFER AREA BEFORE AND AFTER THE SYSTEM IMPROVED

\begin{tabular}{|c|c|c|}
\hline & $\begin{array}{c}\text { Output result } \\
\text { before the system } \\
\text { improved }\end{array}$ & $\begin{array}{c}\text { Output result after the } \\
\text { system improved }\end{array}$ \\
\hline Maximum stay time & $221.5 \mathrm{~s}$ & $129.8 \mathrm{~s}$ \\
\hline Average stay time & $53.6 \mathrm{~s}$ & $19.7 \mathrm{~s}$ \\
\hline $\begin{array}{c}\text { No-load time } \\
\text { percentage }\end{array}$ & $28.0 \%$ & $10.7 \%$ \\
\hline $\begin{array}{c}\text { Congestion time } \\
\text { percentage }\end{array}$ & $31.0 \%$ & $11.2 \%$ \\
\hline Unloading quantity & 403 & 629 \\
\hline
\end{tabular}

Observing the simulation running situation of the model, combining with the result of Figure 16 and Table 1 , and contrasting the traditional storage mode with the optimized storage mode, after adopting the optimized storage mode, the storage location assignment optimization model consumes lower energy and takes shorter time, improves the loading-unloading efficiency, shortens the stacker's operating distance. It gets satisfying result.

\section{CONCLUSIONS}

Based on analyzing the storage location assignment strategy of AS/RS, this paper has constructed the mathematical model on the storage location assignment in AS/RS, and then optimized and solved it. Meanwhile,

Guiliang Zhou (Huaian, Jiangsu; 1982.01), received the Bachelor of Transportation Management from the Changsha University of Science \& Technology, Changsha, P. R. China in 2005. He received the Master of Transportation Planning and Management from the Wuhan University of Technology, Wuhan, P. R. China in 2007.

$\mathrm{He}$ is currently lecturer of the Huaiyin Institute of Technology. His main research interests include logistics simulation, warehouse optimization and logistics equipment. He has published more than 10 papers, 6 were indexed by EI. it has built the simulation model of the storage location assignment in AS/RS by Flexsim, given out the procedure and method of the simulation analysis. Through the simulation experience and analysis it has proved that the optimized storage location assignment model can solve the storage location assignment problem in AS/RS efficiently, and improve the loading-unloading operation efficiency. The shortage of this paper is that the factors taken into account are incomprehensive when constructing the storage location assignment model and the method to solve the model needs further improvement and optimization.

\section{ACKNOWLEDGMENT}

The authors express heartfelt acknowledgment to the experts who have reviewed and evaluated the paper for giving sincere and valuable advices.

\section{REFERENCES}

[1] ZHU Yanru, ZHOU Guiliang and MAO Lina. "Design of Intelligent Decision Module for Storage Location Assignment in AS/RS," The 9th International Conference of Chinese Transportation Professionals, vol. 1, Aug. 2009 , PP. 3118-3123

[2] ZHOU Guiliang and MAO Lina, “The Module Design of Intelligent Allocating Storage Space in Container Freight Station," First International Workshop on Database Technology and Applications, vol. 1, Apr. 2009, PP. 333336

[3] ZOU Huihua. A Study of Optimization for Goods2Placement Distribution Strategies. Journal of Hubei University of Technology. Jun. 2008,pp.43-45

[4] JI Shouwen. Research on Simulation and Optimization of Warehouse System Based on Flexsim Software. Logistics Technology. Oct. 2009,pp.72-74

[5] CHEN Yue-ting.Location Assignment Optimization of AS/RS Based on Genetic Algorithm. Logistics Sci- Tech. Jun. 2008,pp.38-41
Lina Mao (Huaian, Jiangsu; 1982.01), received the Bachelor of Transportation Management, Master of Transportation Planning and Management from the Wuhan University of Technology, Wuhan, P. R. China in 2004 and 2006 respectively.

She is currently lecturer of the Huaiyin Institute of Technology. Her main research interests include supply chain management and warehouse optimization. She has published 6 papers, 4 were indexed by EI. 